Open Access Full Text Article

\title{
Interactive Network Platform Improves Compliance and Efficacy of Subcutaneous Immunotherapy for Patients with Allergic Rhinitis
}

This article was published in the following Dove Press journal:

Patient Preference and Adherence

\author{
Zhiying Shen' \\ Guolin $\operatorname{Tan}^{2}$ \\ Zhuqing Zhong' \\ Siqing Ding' \\ Fang Wang ${ }^{2}$
}

'Nursing Department, Third Xiangya Hospital, Central South University, Changsha, People's Republic of China;

${ }^{2}$ Department of Otolaryngology Head Neck Surgery, Third Xiangya Hospital, Central South University, Changsha,

People's Republic of China
Correspondence: Fang Wang Department of Otolaryngology Head Neck Surgery, Third Xiangya Hospital, Central South University, I38 Tongzipo Road, Changsha 410013, People's

Republic of China

$\mathrm{Tel} / \mathrm{Fax}+8673 \mathrm{I}-88618624$

Email wangfang_xy3yy@163.com
Background: Allergic rhinitis (AR) is a common chronic inflammatory disease of nasal mucosa worldwide, and its symptoms seriously affect the lives of patients. Subcutaneous immunotherapy (SCIT) is an effective treatment for AR, but it is also associated with low patient compliance and difficulties in fully achieving therapeutic effects.

Objective: In this prospective randomized controlled study, we verified the effects of an interactive network platform named "U breath" in improving patient compliance and the efficacy of SCIT in patients with AR.

Methods: A total of 148 patients who received SCIT were recruited as participants and randomly assigned to either the standardized management (SM) or the interactive network platform management (INP) group. The SM group experienced the standard management SCIT process. The INP group experienced a new management approach based on an interactive network platform called "U breath". The compliance rate, combined symptom and medication score (CSMS), visual analogue scale score and the rhinoconjunctivitis quality of life questionnaire (RQLQ) results were evaluated at baseline and 1-year postintervention for the two groups. Results: Within the first year of treatment, the INP group had a higher compliance rate than did the SM group, with a statistically significant difference $(\mathrm{P}<0.05)$. The INP group showed better clinical improvement than the SM group did in terms of the VAS score, and the RQLQ score except the sleep problems $(\mathrm{P}<0.05)$.

Conclusion: This study confirmed that the application of an interactive network platform is of great significance for improving patient compliance and the treatment effects of SCIT in patients with AR.

Keywords: allergic rhinitis, subcutaneous immunotherapy, compliance, quality of life, network, management

\section{Introduction}

Currently, allergen-specific immunotherapy (AIT) is the only treatment available that can prevent the natural progression of allergic rhinitis (AR). ${ }^{1}$ Subcutaneous immunotherapy (SCIT) is one of the main methods of implementing AIT, and it has been shown to have the potential to prevent the further development of allergic sensitization and asthma in patients with $\mathrm{AR} .^{2,3}$ To ensure the long-lasting clinical effects following the end of treatment, the SCIT treatment duration should be at least 3 years. ${ }^{4}$ The effectiveness of SCIT is largely influenced by patient compliance with this therapy. ${ }^{5}$ However, many studies have shown that the level of compliance with SCIT is not satisfactory. In a study by Manzotti et al, the drop-out rate reached $15.6 \%$ in the 
first year of SCIT. ${ }^{6}$ In a retrospective study of 2,796 patients who received SCIT, $37 \%$ of the patients were late for treatment at least once during the three-year course, $20 \%$ dropped out in the first year, and only $23 \%$ completed the three-year course. ${ }^{7}$ Lemberg et al analysed the compliance of 207 SCIT patients at private allergy centres in Germany and found that the overall drop-out rate was $32.4 \%$ over the 3 -year treatment period, and the first year of the treatment period was considered a high-risk period during which patients usually chose to quit. ${ }^{8}$ The consequences of noncompliance are concerning because noncompliance can tremendously affect the effectiveness of SCIT and lead to more medical expenses for patients. $^{7,9}$

Achieving adequate symptom control is pivotal to the success of AR management and the main expectation of patients. ${ }^{10,11}$ The scientific and systematic management of patients undergoing SCIT administered by professional medical personnel may contribute to improving patients' treatment compliance to achieve the optimal therapeutic effect. ${ }^{12,13}$ However, studies have shown that the majority of doctors or nurses do not pay much attention to patient management during treatment. ${ }^{14,15}$ A lack of effective communication between health care providers and patients leads to inadequate disease control, noncompliance, and dissatisfaction in some patients. ${ }^{16}$

With the popularization of computers and smart phones as well as the development of network technology, interactive network tools with powerful data collection, analysis, processing and real-time communication functions may be valuable for SCIT patient management. Therefore, the aim of this study was to examine the effects of an interactive network platform in improving the compliance of patients with AR and the efficacy of SCIT. Compliance was the primary outcome of interest. Other outcomes, such as the combined symptom and medication score, the visual analogue scale score and the rhinoconjunctivitis quality of life questionnaire results, were also examined.

\section{Methods}

\section{Participants and Randomization}

In this prospective randomized controlled trial, participants were assigned to a standardized management (SM) or an interactive network platform management (INP) group, and 12 months of follow-ups from the intervention date were carried out between January and June 2018 (Clinical trial registration number: ChiCTR1900026489).
The patients with a confirmed diagnosis of AR according to the Chinese guidelines for the diagnosis and treatment of allergic rhinitis were recruited from the Department of Otolaryngology Head and Neck Surgery in the participating academic institutions. ${ }^{17}$ Participants were included if they (1) were $\geq 18$ years of age; (2) were prescribed SCIT according to the guidelines and the physician's assessment and did not have any contraindications for SCIT; and (3) were proficient in using smartphones and had a smartphone. Participants were excluded if they (1) experienced a severe systemic reaction (rapid onset $(<15$ mins), widespread urticaria, angioedema or severe asthma) or anaphylactic shock of unknown origin during treatment; (2) were participating in other projects related to SCTI management; or (3) were suffering from other serious physical or psychological conditions, such as cancer, chronic kidney failure, schizophrenia, and autism.

The sample size was calculated on the basis of a retrospective analysis of data from all of the patients treated with SCIT at the medical centre during 2017. Among a total of 482 patients, $75 \%$ had good treatment compliance in the first treatment year. With the integrated effect size of the patient compliance to the interactive network platform intervention set at $20 \%$, the significance level set at 0.05 , and the test power set at 0.9 , the optimal sample size was 62 . Considering a withdrawal rate of $20 \%$, the final sample size was 74 in each group. Individual patients were divided into the SM or INP groups using the random-number generator function in SPSS. The grouping situation was concealed from the data collectors. A total of 148 eligible participants who underwent SCIT were recruited, with 74 cases in each group.

\section{Therapeutic Regimen}

All patients in the study were treated with standard SCTI by trained otolaryngology nurses in accordance with the "clinical practice specifications for subcutaneous immunotherapy for allergic rhinitis"18 issued by the Chinese Allergic Rhinitis Research Collaboration Group. Both groups were treated with a conventional schedule with increasing doses of allergen immunotherapy extract administered once a week, and the vaccine brand used was ALUTARD (house dust mite allergen, produced by Alk-Abello A/S). According to the drug instructions, the total course of treatment is generally 3 years and includes two stages. The first stage was the initial (dose accumulation) phase, which usually lasted 14-15 weeks with one injection per week. When the maximum tolerated dose reached 100,000 sq-u, 
the second phase (maintenance phase) began. Injections were administered every 4-6 or 4-8 weeks during the phase. All injection procedures for the patients were performed by the same nurse who was blinded to which group the patient was in to reduce the bias caused by the operator during the intervention operation.

\section{Compliance Rate}

Compliance rate $=$ number of compliant people/total number of people $\times 100 \%$.

Based on the instructions provided by ALUTARD, in the initial stage, the doses were adjusted if the intervals between any two injections were less than 2 weeks or more than 3 weeks. During the maintenance phase, the doses were adjusted if the patient did not receive the injection for more than 8 weeks. Therefore, if the doses were adjusted for any of the above mentioned reasons for a patient, the patient was considered noncompliant. Any participants who expressed an intention to withdraw from SCIT were also regarded as noncompliant.

\section{Combined Symptom and Medication Score (CSMS)}

The CSMS is an important indicator recommended by the European Academy of Allergy and Clinical Immunology (EAACI) Immunotherapy Interest Group to evaluate the clinical effect of AIT in AR patients. ${ }^{19}$ CSMS is composed of daily symptom score (dSS) and daily medication score (dMS). (1) The dSS consists of four nasal symptoms of AR (itchy nose, sneezing, runny nose, blocked nose) and two conjunctival symptoms (itchy/red eyes, watery eyes). The score ranges from $0-3$ points for each item: $0=$ no symptoms; 1= mild symptoms; $2=$ moderate symptoms; and $3=$ severe symptoms. The overall symptom score ranged from $0-18$, and the dSS was equal to the total symptom score divided by 6. (2) The dMS was determined based on the World Allergy Organization recommendations for a stepwise approach in administering rescue medication: $1=$ oral and/or topical (eyes or nose) nonsedative $\mathrm{H} 1$ antihistamines $(\mathrm{H} 1 \mathrm{~A}) ; 2=$ intranasal corticosteroids (INS) with/without H1A; and 3=oral corticosteroids with/ without INS and with/without H1A. The CSMS (0-6)= dss $(0-3)+\mathrm{dMS}(0-3)$.

\section{Visual Analogue Scale (VAS)}

The VAS is a psychometric response scale for assessing the overall discomfort of AR.
Patients rank their symptoms by drawing a vertical line on a $10-\mathrm{cm}$ straight line representing severity from 0 : 'not at all bothersome' to 10 : "extremely bothersome". ${ }^{20,21}$

\section{Rhinoconjunctivitis Quality of Life Questionnaire (RQLQ)}

The RQLQ is an instrument with 28 questions that is used to assess adult patients (aged from 17 to 70 ) with rhinoconjunctivitis. $^{22}$ The RQLQ covers 7 domains (activity limitations, sleep problems, nose symptoms, eye symptoms, non-nose/eye symptoms, practical problems and emotional function). The patients recalled how bothered they have been due to rhinoconjunctivitis during the previous week with regard to each question on a 7-point scale $(0=$ not impaired at all, $6=$ severely impaired). The total RQLQ score was the mean score of all 28 responses, and the mean score of all the items in each domain was considered the domain score. The Cronbach's alpha of the Chinese version of the RQLQ scale was 0.947 , the Cronbach's alpha of each domain was $0.834,0.827,0.918$, $0.805,0.808,0.791$, and 0.853 , respectively.

\section{Data Collection}

Before the intervention, the following sociodemographic and clinical characteristics were collected from each participant: sex, age, education level, and the presence/absence of other allergic diseases, a family history of AR and a history of smoking. Compliance was assessed using the compliance rate, and the therapeutic effects were assessed using the CSMS, the RQLQ and the VAS scores at the beginning of the intervention and at 12 months postintervention.

\section{Data Analysis}

All data were analysed using SPSS 24.0 (IBM Corp., Armonk, NY, USA). Descriptive statistics (means, standard deviations, and percentages) were used to characterize the participants according to the demographic data and disease and treatment information. The independent $t$-test, Pearson's $\chi 2$-test, and the Wilcoxon rank-sum test were used to evaluate the differences in the sociodemographic and clinical characteristics and the scores of the CSMS, VAS, and RQLQ between the SM group and INP group. The $\chi 2$-test was used to compare the compliance rate between the two groups. A two-tailed test was performed at a 0.05 significance level. 


\section{Standardization Management Group (SM Group)}

The standardized SCIT management procedure issued by the Chinese Allergic Rhinitis Research Collaboration Group was adopted by the present study, and the main operations were as follows (Figure 1).

1. Personal files were created for patients, and the content of the personal files included a. demographic and disease data; $b$. injection information, including the time, site of injection, bottle number, dose $(\mathrm{mL})$, adverse reactions, treatment methods and treatment outcomes after a reaction occurred; and c. follow-up information, including the follow-up dates and the CSMS, VAS, and RQLQ scores.

2. Injection record cards for patients were distributed. The information on the record card included the patient's name, the time of each injection and injection site, and the time of the next injection, and the patients were required to carry the card with them during each treatment.

3. Health education was provided. The patients and their families attended a $30 \mathrm{~min}$ face-to-face health education course led by two otolaryngologic nurses who had received standardized SCIT training, and the patients and their families received a health education manual. The contents of health education course and manual primarily included the knowledge of AR, features of SCIT, issues requiring attention during SCIT, adverse reactions and coping methods of SCIT, as well as how to deal with environment factors and how to better self-manage their disease.

4. Follow-up: If a patient failed to receive treatment within 7 days of the prescribed injection time, the outpatient nurse reminded the patient by phone or a text message.

\section{Interactive Network Platform Management (INP Group)}

In collaboration with a medical technology company, the researchers in the present study designed an interactive network platform called "U breath" for SCIT patients and health care providers, which can be used on computers and smartphones. "U breath" includes a medical staff side and the patient side, through which the medical staff and patients can interact and communicate with one another. The main functions of "U breath " are as follows (Figure 2).

(1) Establishment of electronic personal profile. Patients filled in a form to apply for the SCIT treatment plan online, and the medical staff established health records for the patients after the examination and approval. (2) Automatic reminder. To avoid "information fatigue" in patients, "U breath" only sent a reminder message the day before and the day after the injection. The first message informed patients that they needed to receive treatment the

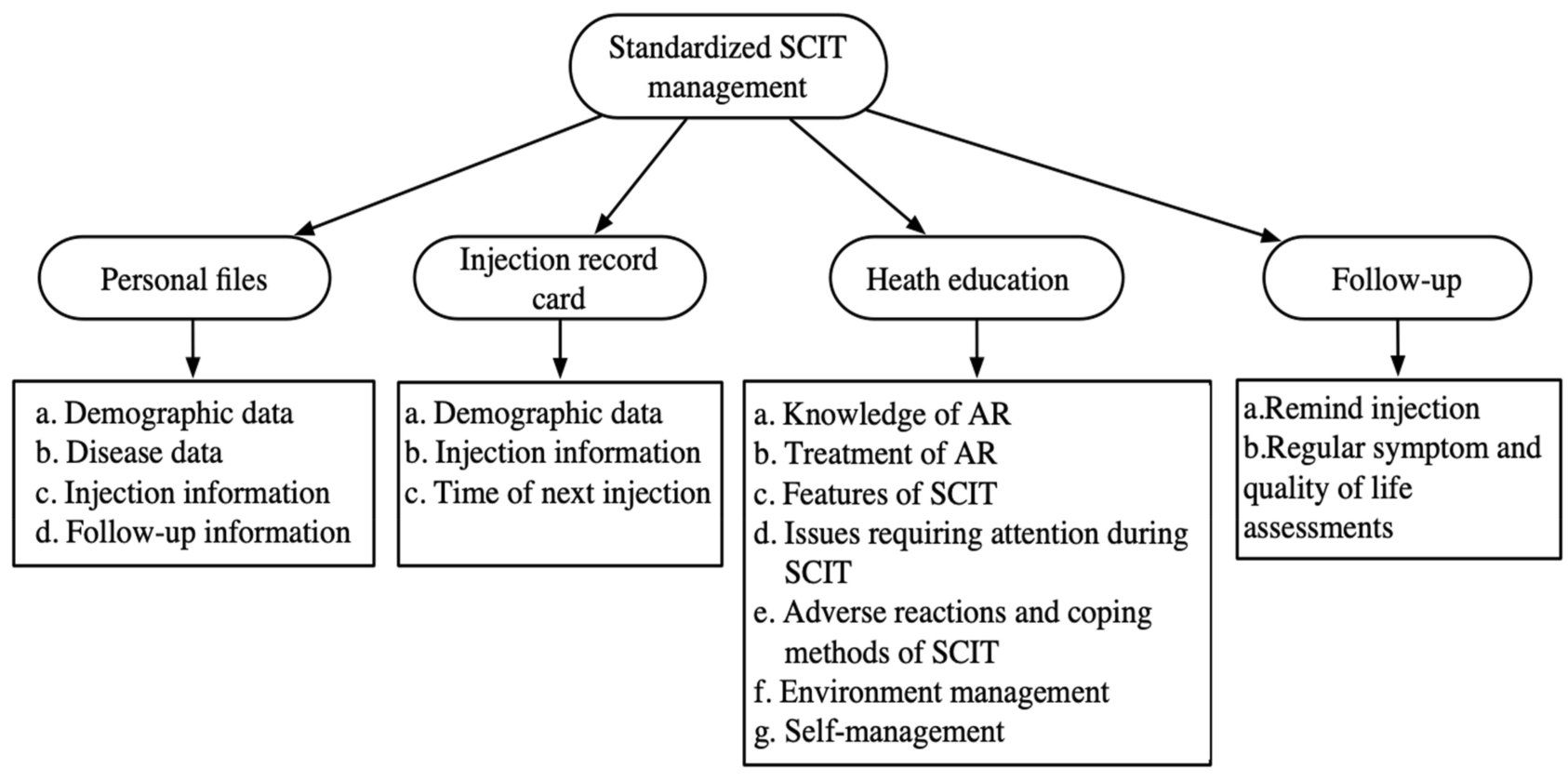

Figure I Content of standized SCIT management (SM group). 


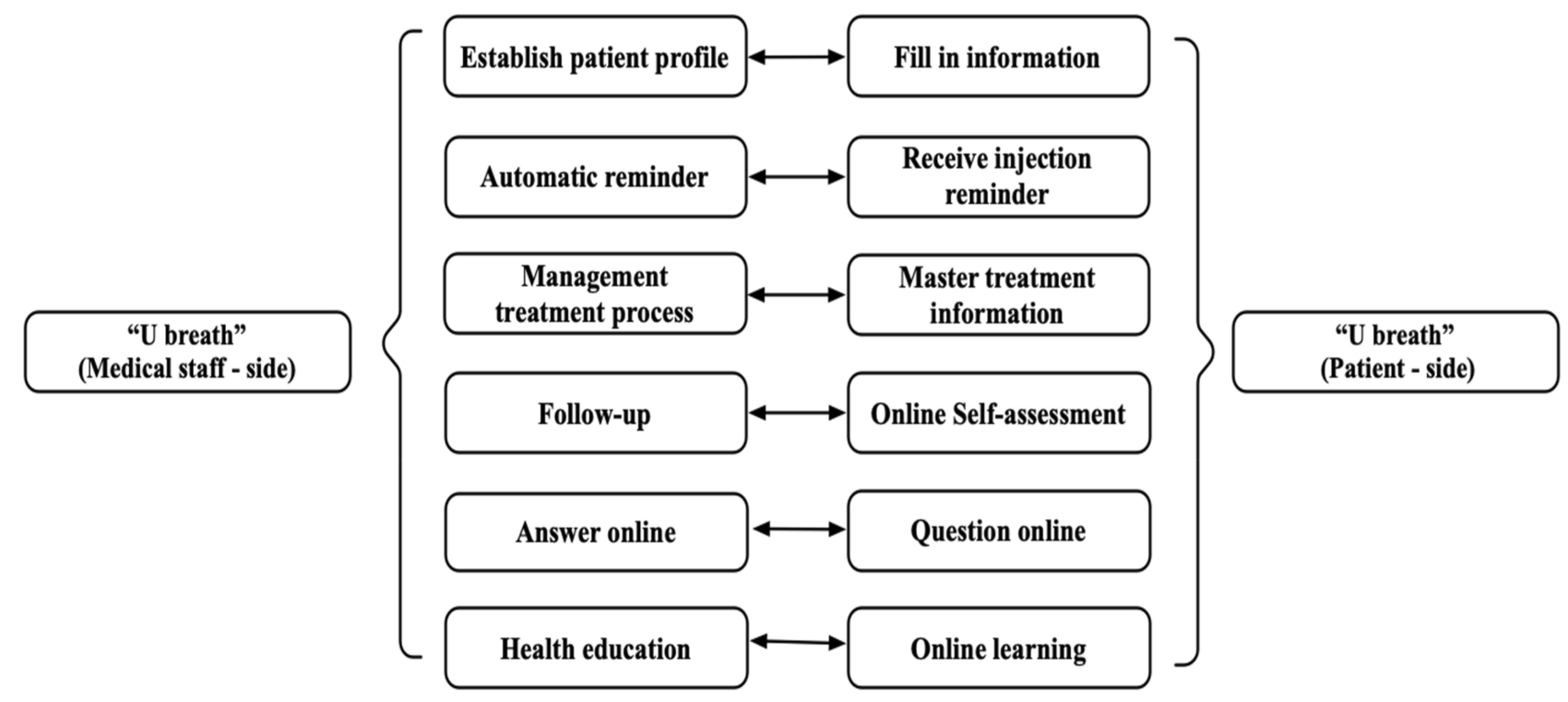

Figure 2 Interaction of functions between medical staff-side and patient-side.

next day, and the message had two options, "yes" or "no", so patients could choose whether to receive treatment at the scheduled time according to their current state. The purpose of the second message was to inform the patient of the time of the next injection and the precautions to take after the injection. In addition, the patient's shedding tendency was evaluated according to the injection status (shedding tendency: the initial period was more than 2 weeks, and the maintenance period was more than 8 weeks without treatment). For patients with a shedding tendency, the nurse sent reminder messages through the platform in a timely manner. (3) Management of treatment process. On this platform, the medical staff inquired all patients who received SCIT about their treatment stage and identified the patients who needed treatment within the next week, which helped the medical staff to prepare and arrange the manpower and material resources needed for the outpatient services. Accordingly, patients could also query their own treatment information on the "patient side". (4) Follow-up. "U breath" automatically sent electronic versions of the RQLQ and VAS scales to patients every two months. After patients filled them in, the platform sent the results to doctors and nurses. (5) Online consultations and responses. If patients had questions about the treatment, they asked for answers online, and the medical staff replied to them timely. (6) Health education. Although the health education content was basically the same as that in the manual provided to the SM group, "U breath" presented health education in the form of pictures and videos, which were intended to help patients better understand the information without needing any medical knowledge.

\section{Results}

\section{Sample Characteristics}

In total, 192 participants were screened for inclusion, 148 (77.1\%) of whom met the inclusion criteria and consented to participate in this study, and all of them were divided at random. Six participants (4.1\%) did not complete the intervention. Four patients living in another city requested to take the remaining drugs back to the local medical institution after the first injection at the hospital, so the compliance of these patients and the effectiveness of the treatment for these patients could not be tracked; one patient was lost to follow-up, and one patient declined to continue (Figure 3). There were 72 members in the INP group and 70 in the SM group. No significant differences were found between the two groups in terms of the sociodemographic and clinical characteristics (Table 1).

\section{Comparison of the Compliance Rate and Cause Analysis Between the INP and SM Groups}

During the year after the intervention, a total of 23 (16.2\%) patients did not receive treatment on schedule, and the compliance rate in the INP group was $90.3 \%$, which was significantly higher than that in the SM group (77.1\%) $(\mathrm{P}=0.034)$. In both groups, the main reason for 


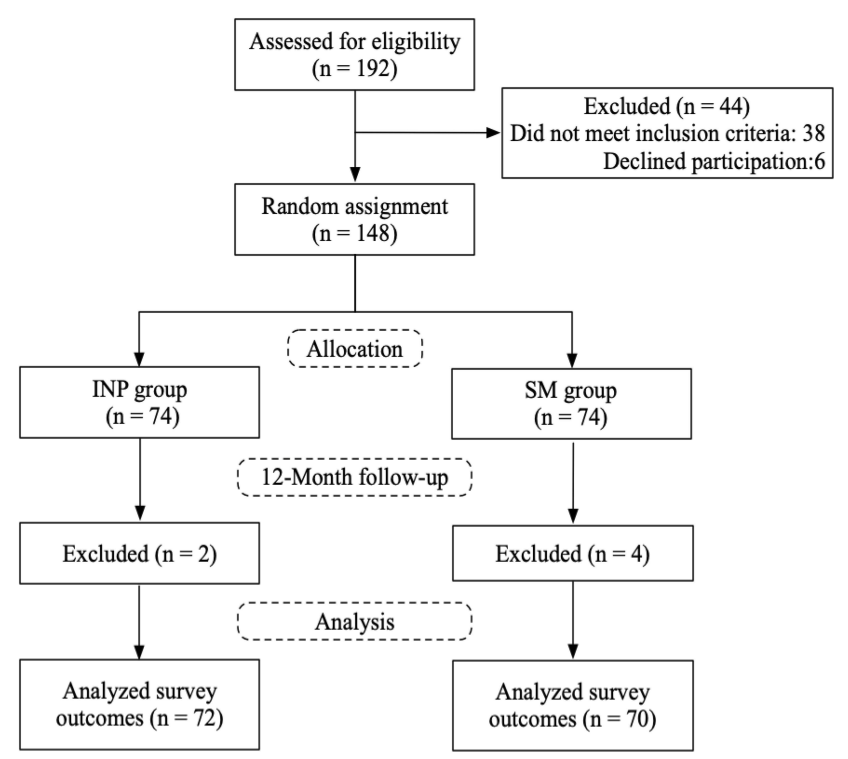

Figure 3 Study flow diagram.

patient noncompliance was the high cost of treatment (INP group $3(4.2 \%)$ vs SM group $4(5.7 \%))$ and the long duration of treatment (INP group $2(2.8 \%)$ vs SM group $5(7.1 \%))$. The second reason was that due to the therapeutic effect of SCIT may not be obvious in the short term, so patients lost confidence in treatment (INP group 1 $(1.4 \%)$ vs SM group $3(4.3 \%))$. Other reasons were that patients' self-perceived symptoms improved significantly after a certain period of time during the treatment, so those patients thought that there was no need to continue treatment (INP group $0(0.0 \%)$ vs SM group $3(4.3 \%)$ ), and some patients terminated the treatment due to adverse reactions (INP group $1(1.4 \%)$ vs SM group $1(1.4 \%)$ ). We did not observe significant differences in the reasons for noncompliance between the two groups $(\mathrm{P}>0.05)$ (Table 2).

\section{Comparison of the VAS and CSMS Scores Between the INP and SM Groups}

The difference in the VAS and CSMS scores between the two groups before intervention was not statistically significant $(\mathrm{P}>0.05)$. At 12 months, the INP group had a score of 3.0 and the SM group had a score of $3.79(\mathrm{P}=0.003)$ on the VAS, and on the CSMS, the scores were 1.44 and 1.83 , respectively; significant differences were identified $(\mathrm{P}=0.002)$ (Table 3). Moreover, the INP group had a score of 0.66 on the dSS and a score of 0.96 on the dMS, and the SM group had a score of $0.88(\mathrm{P}=0.001)$ and a score of $0.96(\mathrm{P}=0.034)$ on these measures, respectively (Table 3 ).

\section{Comparison of the RQLQ Between the INP and SM Groups}

Before the intervention, there was no significant difference in the scores for the 7 dimensions and the total scores of

Table I Sample Characteristics $(n=148)$

\begin{tabular}{|c|c|c|c|c|}
\hline Variable & INP Group $(n=74)$ & SM Group $(n=74)$ & t $/ \chi^{2}$ Value & P-Value \\
\hline \multicolumn{5}{|l|}{ Sex,N(\%) } \\
\hline Male & $42(56.76)$ & $40(54.05)$ & 0.109 & $0.74 I$ \\
\hline Female & $32(43.24)$ & $34(45.95)$ & & \\
\hline Age,year mean(SD) & $25.32(5.62)$ & $25.89(6.04)$ & 0.594 & 0.553 \\
\hline \multicolumn{5}{|l|}{ Education level,N(\%) } \\
\hline Secondary school or below & $39(52.70)$ & $45(60.8 I)$ & 0.991 & 0.320 \\
\hline Bachelor degree or above & $35(47.30)$ & $29(39.19)$ & & \\
\hline \multicolumn{5}{|l|}{ Complication with other allergic diseases } \\
\hline Yes & $28(37.84)$ & $25(33.78)$ & 0.265 & 0.607 \\
\hline No & $46(62.16)$ & $49(66.22)$ & & \\
\hline \multicolumn{5}{|l|}{ Family history of AR } \\
\hline Yes & $35(47.30)$ & $32(43.24)$ & 0.245 & 0.620 \\
\hline No & $39(52.70)$ & $42(56.76)$ & & \\
\hline \multicolumn{5}{|l|}{ History of smoking } \\
\hline Yes & $14(18.92)$ & $17(22.97)$ & 0.367 & 0.545 \\
\hline No & $60(81.08)$ & $57(77.03)$ & & \\
\hline
\end{tabular}

Abbreviations: INP, Interactive network platform management; SM, Standardized management; AR, Allergic rhinitis. 
Table 2 Rate of Noncompliance and Cause Analysis

\begin{tabular}{|c|c|c|c|c|c|}
\hline Causes of Non-Compliance & $\begin{array}{l}\text { Total Number of Two } \\
\text { Groups }(n=\mid 42)\end{array}$ & INP Group $(n=72)$ & SM Group $(n=70)$ & $\chi^{2}$ Value & $P$-value \\
\hline Number of total non-compliance & $23(16.2)$ & $7(9.7)$ & $16(22.9)$ & 4.511 & 0.034 \\
\hline High cost of treatment & $7(4.9)$ & $3(4.2)$ & $4(5.7)$ & & \\
\hline Long course of treatment & $7(4.9)$ & $2(2.8)$ & $5(7.1)$ & & \\
\hline No obviously curative effect & $4(2.8)$ & $\mathrm{I}(\mathrm{I} .4)$ & $3(4.3)$ & & \\
\hline Symptom improvement & $3(2.1)$ & $0(0.0)$ & $3(4.3)$ & & \\
\hline Adverse reactions & $2(1.4)$ & $\mathrm{I}(\mathrm{I} .4)$ & $\mathrm{I}(\mathrm{I} .4)$ & & \\
\hline
\end{tabular}

Table 3 Differences in VAS and CSMS Scores Between the Two Groups

\begin{tabular}{|c|c|c|c|c|c|c|c|c|}
\hline \multirow[t]{2}{*}{ Measures } & \multicolumn{4}{|l|}{ Baseline(SD) } & \multicolumn{4}{|c|}{ I 2 Month Follow Up(SD) } \\
\hline & $\begin{array}{l}\text { INP Group } \\
(\mathbf{N}=72)\end{array}$ & $\begin{array}{l}\text { SM Group } \\
(\mathrm{N}=70)\end{array}$ & $t$ value & $P$-value & $\begin{array}{l}\text { INP Group } \\
(\mathbf{N}=72)\end{array}$ & $\begin{array}{l}\text { SM Group } \\
(\mathrm{N}=70)\end{array}$ & $t$ value & $P$-value \\
\hline VAS & $6.83(1.83)$ & $6.73(1.76)$ & 0.332 & $0.74 I$ & $3.00(1.38)$ & $3.79(I .7 I)$ & 3.034 & 0.003 \\
\hline CSMS & $3.85(0.88)$ & $3.75(0.77)$ & 0.719 & 0.473 & $1.44(0.73)$ & $1.83(0.7 \mathrm{I})$ & 3.226 & 0.002 \\
\hline dSS & $1.63(0.64)$ & $1.60(0.47)$ & 0.317 & 0.751 & $0.66(0.36)$ & $0.88(0.41)$ & 3.400 & 0.001 \\
\hline $\mathrm{dMS}$ & $2.22(0.5 \mathrm{I})$ & $2.14(0.52)$ & 0.926 & 0.356 & $0.78(0.5 \mathrm{I})$ & $0.96(0.49)$ & 2.144 & 0.034 \\
\hline
\end{tabular}

Abbreviations: VAS, Visual analogue scale; CSMS, Combined symptom and medication score; dSS, daily symptom score; dMS, daily medication score.

the RQLQ between the two groups ( $\mathrm{P}>0.05)$ (Table 4). However, after 1 year, the INP group had a score of 1.96 on activity limitations, 1.24 on sleep problems, 1.00 on non-nose/eye symptoms, 1.55 on practical problems, 1.31 on nasal symptoms, 0.47 on eye symptoms, and 0.83 on emotional function, and the SM group had scores of 2.57 $(\mathrm{P}=0.000), \quad 1.43 \quad(\mathrm{P}=0.129), \quad 1.38 \quad(\mathrm{P}=0.000), \quad 2.02$ $(\mathrm{P}=0.014), \quad 1.60 \quad(\mathrm{P}=0.043), 0.67 \quad(\mathrm{P}=0.024)$, and 1.26 $(\mathrm{P}=0.000)$ on these dimensions, respectively (Table 4). The posttreatment total RQLQ score and the other RQLQ domain scores were significantly lower in the INP group than in the SM group $(\mathrm{P}<0.05)$, with the exception of the sleep problems.

\section{Discussion}

In this study, an interactive network platform intervention was provided for patients who were treated with SCIT to improve their compliance and the efficacy of the treatment. The primary hypothesis that a bidirectional, integrated management program based on interactive network platforms would significantly improve treatment compliance in SCIT users was supported. One year after using " $U$ breath", the noncompliance rate of the INP group was $9.7 \%$, which was significantly lower than that of the SM group (22.9\%).

SCIT has a long treatment period and is characterized by a high cost and a slow onset. In addition, it may lead to serious adverse reactions, which were also important

Table 4 Differences in RQLQ Scores Between the Two Groups

\begin{tabular}{|c|c|c|c|c|c|c|c|c|}
\hline \multirow[t]{2}{*}{ Measures } & \multicolumn{4}{|c|}{ Baseline(SD) } & \multicolumn{4}{|c|}{ I 2 Month Follow Up(SD) } \\
\hline & $\begin{array}{l}\text { INP Group } \\
(\mathrm{N}=\mathbf{7 2})\end{array}$ & $\begin{array}{l}\text { SM Group } \\
(\mathrm{N}=70)\end{array}$ & $t$ value & $P$-value & $\begin{array}{l}\text { INP Group } \\
(\mathbf{N}=\mathbf{7 2})\end{array}$ & $\begin{array}{l}\text { SM Group } \\
(\mathrm{N}=70)\end{array}$ & $t$ value & $P$-value \\
\hline Activity & $3.23(1.16)$ & $3.24(1.03)$ & 0.434 & 0.665 & $1.96(0.84)$ & $2.57(0.93)$ & 4.104 & 0.000 \\
\hline Sleep & $2.00(1.23)$ & $1.84(1.24)$ & 0.772 & $0.44 I$ & $1.24(0.68)$ & $1.43(0.80)$ & 1.526 & 0.129 \\
\hline Non-nose/eye symptoms & $2.0 \mathrm{I}(0.77)$ & $1.91(0.70)$ & 0.809 & 0.420 & $1.00(0.42)$ & $1.38(0.5 \mathrm{I})$ & 4.853 & 0.000 \\
\hline Practical & $2.83(I .32)$ & $2.87(1.47)$ & 0.171 & 0.865 & $1.55(0.87)$ & $2.02(1.33)$ & 2.498 & 0.014 \\
\hline Nasal & $3.11(1.29)$ & $3.08(1.26)$ & 0.140 & 0.889 & $\mid .31(0.60)$ & $1.60(1.04)$ & 2.042 & 0.043 \\
\hline Eye & $1.09(0.94)$ & $1.12(0.80)$ & 0.205 & 0.838 & $0.47(0.43)$ & $0.67(0.60)$ & 2.288 & 0.024 \\
\hline Emotions & $2.45(1.28)$ & $2.34(1.15)$ & 0.538 & 0.591 & $0.83(0.5 \mathrm{I})$ & $1.26(0.85)$ & 3.667 & 0.000 \\
\hline Total of RQLQ & $2.31(0.85)$ & $2.26(0.78)$ & 0.365 & 0.716 & $\mathrm{I} .13(0.34)$ & $1.50(0.44)$ & 5.616 & 0.000 \\
\hline
\end{tabular}

Abbreviation: RQLQ, Rhinoconjunctivitis Quality of Life Questionnaire. 
reasons for patient noncompliance. ${ }^{6,23,24}$ Consistent results were identified in the present study from the analysis of the reasons for participant noncompliance. However, most of the reasons that compromised patient compliance with SCIT, except for the overwhelming cost, can be overcome through adequate health education and communication with patients. ${ }^{23,25}$ These aspects can prevent misunderstandings, mistakes and inappropriate expectations; therefore, the level of compliance increased.

A close relationship between allergists and their patients during SCIT had great potential to affect the concordance levels of SCIT. ${ }^{26}$ In Sanchez's ${ }^{27}$ study, health education and follow-ups of patients were strictly carried out by medical staff, and the results showed a significant improvement in patient compliance( $11 \%$ vs $21 \%, \mathrm{P}=0.02)$. Savi et $\mathrm{al}^{28}$ achieved an increased level of compliance by providing educational sessions for patients and conducting regular follow-up with patients by email or telephone. However, in these studies, instantaneous guidance over long periods of time was difficult to provide to patients at home. In addition, telephones or E-mail were often used for the follow-up visits, which are inefficient and cumbersome; not only is a substantial amount of time required for medical staff to read patients' medical records and to remind each patient by a phone call, but it is also difficult to encourage and motivate patients to seek medical resources.

With the rapidly growing numbers of mobile Internet users, smartphones and laptops have inevitably become key components of patients' self-management tools. In Wu's study, ${ }^{29}$ the application of social networking software(We Chat) improved the compliance of patients receiving subcutaneous immunotherapy. The results of this study also demonstrate the potential value of network technology in improving patients' behavior. First, in this study, the "U breath" provided a timely and efficient support platform for health education and doctor-patient communication, and the health education materials provided in the electronic platform were in the form of pictures and videos enclosed with text explanations, making it easier for patients to receive and understand. Second, the "U breath" had an automatic reminder function, which automatically sent an SMS to remind patients after the previous injection and before the next injection. In addition, it could identify which patients have the tendency to be noncompliant so that medical staff could receive an early warning and prevent the occurrence of lateness or withdrawal. Finally, the intelligent patient management system could improve the work efficiency of medical staff, reduce the burden and save medical resources.
Suboptimal control of symptoms had a serious impact on the quality of life of patients with AR. The patients were embarrassed and depressed by their symptoms, and they even consistently exhibited a significant decline in cognition, learning and memory. ${ }^{30}$ As a result, health care professionals need to be concerned not only about patients' adherence to medication or SCIT but also about the status of their symptoms and their quality of life. ${ }^{25}$ Cemal et $\mathrm{al}^{31}$ used a mobile phone software named "physician on call patient engagement trial" in 88 patients with $\mathrm{AR}$, and the results showed that the quality of life of AR patients and the health outcomes improved significantly.

In the present study, "U breath" helped patients and medical staff to comprehensively manage AR patients with the treatment of SCIT, as the platform built an efficient communication bridge between patients and health service providers. Patients not only know what to do but also why and how to deal with AR through this platform. The results of this study showed that after 1 year of the intervention, the VAS, CSMS and RQLQ scores of patients in the INP group were lower than those in the SM group $(P<0.05)$, indicating that significantly more symptom remission occurrences and better treatment effects were identified in the INP group.

\section{Study Limitations}

There were some limitations in this study. First, provided that children and elderly individuals may not be proficient in using smartphones or electronic devices, we only included adults over the age of 18 as the study subjects. However, future research can also be conducted on children and elderly individuals to improve their disease and symptom management. Second, due to the limitations of human and material resources that could be offered in the present study, our intervention lasted only 1 year; therefore, additional studies focusing on persistent monitoring may still be needed to better evaluate the long-term effects of this intervention. Third, this study was an experimental study with a relatively small number of patients in a single centre, and more large-scale, multi-centre studies are warranted.

\section{Conclusion}

In conclusion, this study proved that the application of an interactive network platform, named "U breath", was of great significance for improving patient compliance and the treatment effects of SCIT on users. In the future, longterm and large-scale studies are needed to further prove the application value of network interactive platforms in the management of patients with AR. 


\section{Ethical Consideration}

The study was reviewed and approved by the ethics committee of the Third Xiangya Hospital of Central South University (no.2015-S082). All the patients who participated in the study signed the informed consent in person. The study was conducted in compliance with the guidelines of the Declaration of Helsinki.

\section{Data Sharing Statement}

All of the individual participant data collected during the trial will be shared. However, the study protocol and clinical study report are not available. Data are available beginning 6 months and ending 12 months after publication. Investigators whose proposed use of the data has been approved by an independent review committee identified for this purpose. Data are available for 6 months at a thirdparty website: http://pan.baidu.com/share/manage.

\section{Acknowledgements}

The authors would like to acknowledge all patients who participated in the study for their support of the study.

\section{Funding}

The program was supported by the Science and Technology Project of Hunan Province (2017SK2043) and the National Natural Science Foundation of China (81870708).

\section{Disclosure}

The authors report no conflicts of interest in this work.

\section{References}

1. Bousquet J, Lockey R, Malling H. Allergen immunotherapy: therapeutic vaccines for allergic diseases. A WHO position paper. J Allergy Clin Immunol. 1998;102(4 Pt 1):558-562. doi:10.1016/s0091-6749(98)70271-4

2. Huang Y, Wang C, Cao F, et al. Comparison of long-term efficacy of subcutaneous immunotherapy in pediatric and adult patients with allergic rhinitis. Allergy Asthma Immunol Res. 2019;11(1):68-78. doi:10.4168/aair.2019.11.1.68

3. Asaria M, Dhami S, Van RR, et al. Health economic analysis of allergen immunotherapy (AIT) for the Management of allergic rhinitis, asthma, food allergy and venom allergy: a systematic overview. Allergy. 2018;73(2):269-283. doi:10.1111/all.13254

4. Okamoto Y, Ohta N, Okano M, et al. Guiding principles of subcutaneous immunotherapy for allergic rhinitis in Japan. Auris Nasus Larynx. 2014;41(1):1-5. doi:10.1016/j.anl.2013.06.003

5. Incorvaia C, Mauro M, Ridolo E, et al. Patient's compliance with allergen immunotherapy. Patient Prefer Adherence. 2008;2(3):247-251. doi:10.2147/PPA

6. Manzotti G, Riario-Sforza GG, Dimatteo M, Scolari C, Makri E, Incorvaia C. Comparing the compliance to a short schedule of subcutaneous immunotherapy and to sublingual immunotherapy during three years of treatment. Eur Ann Allergy Clin Immunol. 2016;48 (6):224-227.
7. Kiel MA, Röder E, Gerth van Wijk R, AI MJ, Hop WC, Rutten-van MMP. Real-life compliance and persistence among users of subcutaneous and sublingual allergen immunotherapy. $J$ Allergy Clin Immunol. 2013;132(2):353-360. doi:10.1016/j

8. Lemberg ML, Berk T, Shahhosseini K, Kasche EM, Mösges R. Sublingual versus subcutaneous immunotherapy: patient adherence at a large German allergy center. Patient Prefer Adherence. 2017;11:63-70. doi:10.2147/PPA.S122948

9. Allen-Ramey F, Mao J, Blauer-Peterson C, Rock M, Nathan R, Halpern R. Healthcare costs for allergic rhinitis patients on allergy immunotherapy: a retrospective observational study. Curr Med Res Opin. 2017;33(11):1-24. doi:10.1080/03007995.20

10. Lipworth B, Newton J, Ram B, Small I, Schwarze J. An algorithm recommendation for the pharmacological management of allergic rhinitis in the UK: a consensus statement from an expert panel. NPJ Primary Care Respir Med. 2017;27(1):3. doi:10.1038/s41533016-0001-y

11. Canonica GW, Triggiani M, Senna GE. 360 degree perspective on allergic rhinitis management in Italy: a survey of GPs, pharmacists and patients. Clin Mol Allergy. 2015;13(1):25. doi:10.1186/s12948015-0029-5

12. Bousquet J, Reid J, van Weel C, et al. Allergic rhinitis management pocket reference 2008. Allergy. 2010;63(8):990-996. doi:10.1111/ j.1398-9995.2008.01642.x

13. Musa F, Al-Ahmad M, Arifhodzic N, Al-Herz W. Compliance with allergen immunotherapy and factors affecting compliance among patients with respiratory allergies. Hum Vaccin Immunother. 2017;13(3):514-517. doi:10.1080/21645515.2016.1243632

14. Ridolo E, Incorvaia C, Senna GE, Montagni M, Olivieri E, Canonica GW. Allergen immunotherapy: clinical and practical education of Italian trainees in allergy and clinical immunology schools. Eur Ann Allergy Clin Immunol. 2013;45(Suppl 2):49-52.

15. Gyuyoung H, Taebum K, Han MY, Dongho N, Jungwon P. A survey of the prescription patterns of allergen immunotherapy in Korea. Allergy Asthma Immunol Res. 2013;5(5):277-282. doi:10.4168/aair.2013.5.5.277

16. Marple B, Fornadley J, Patel A, et al. Keys to successful management of patients with allergic rhinitis: focus on patient confidence, compliance, and satisfaction. Otolaryngol Head Neck Surg. 2007;136(6): S107-S124. doi:10.1016/j.otohns.2007.02

17. Rhinology editorial board of Chin J Otorhinolaryngol Head Neck Surg. Guidelines for diagnosis and treatment of allergic rhinitis (2015, tianjin). Chin J Otorhinolaryngol Head Neck Surg. 2016;51:6-24. doi:10.16066/j.1672-7002.2015.08.001

18. Chinese Allergic Rhinitis Research Collaboration Group. Clinical practice specification for subcutaneous immunotherapy of allergic rhinitis. Chin Arch Otolaryngol Head Neck Surg. 2018;28(1):1-12. doi:10.16066/j.1672-7002.2018.01.001

19. Pfaar O, Demoly P, Wijk R, et al. Recommendations for the standardization of clinical outcomes used in allergen immunotherapy trials for allergic rhinoconjunctivitis: an EAACI position paper. Allergy. 2014;69(7):854-867. doi:10.1111/all.12383

20. Bousquet PJ, Combescure C, Neukirch F, et al. Visual analog scales can assess the severity of rhinitis graded according to ARIA guidelines. Allergy. 2007;62(4):367-372. doi:10.1111/j.1398-9995.2006.01276.x

21. Bousquet PJ, Combescure C, Klossek JM, Daures JP, Bousquet J. Change in visual analog scale score in a pragmatic randomized cluster trial of allergic rhinitis. J Allergy Clin Immunol. 2009;123 (6):1349-1354. doi:10.1016/j.jaci.2009.02.033

22. Juniper EF, Thompson AK, Ferrie PJ, Roberts JN. Validation of the standardized version of the rhinoconjunctivitis quality of life questionnaire. J Allergy Clin Immunol. 1999;104(2):364-369. doi:10.1016/S0091-6749(99)70380-5

23. Egert-Schmidt AM, Kolbe JM, Mussler S, Thum-Oltmer S. Patients' compliance with different administration routes for allergen immunotherapy in Germany. Patient Prefer Adherence. 2014;8:1475-1481. doi:10.2147/PPA.S70326 
24. Leader BA, Rotella M, Stillman L, Delgaudio JM, Patel ZM, Wise SK. Immunotherapy compliance: comparison of subcutaneous versus sublingual immunotherapy. Int Forum Allergy Rh. 2016;6 (5):460-464. doi:10.1002/alr.21699

25. Wadenbäck J, Arnold V. Patient knowledge, perceptions, expectations and satisfaction on allergen-specific immunotherapy: a survey. Respir Med. 2013;107:361-367.

26. Gelincik A, Demir S, Olgaç M, et al. High adherence to subcutaneous immunotherapy in a real-life study from a large tertiary medical center. Allergy Asthma Proc. 2017. doi:10.2500/aap. 2017.38.4091

27. Sánchez J. Adherence to allergen immunotherapy improves when patients choose the route of administration: subcutaneous or sublingual. Allergol Immunopathol. 2015;43(5):436-441. doi:10.1016/ j.aller.2014.04.011
28. Savi E, Peveri S, Senna G, Passalacqua G. Causes of SLIT discontinuation and strategies to improve the adherence: a pragmatic approach. Allergy. 2013;68(9):1193-1195. doi:10.1111/all.12198

29. Wu XP, Qiu RQ, Yang XF, Shen K, Tian ST. Influence on compliance of subcutaneous immunotherapy in patients with allergic rhinitis by We-Media management. J Clin Otorhinol Head Neck Surg. 2018;32 (8):591-594.

30. Blaiss MS. Important aspects in management of allergic rhinitis: compliance, cost, and quality of life. Allergy Asthma Proc. 2003;24 (4):231-238.

31. Cemal C, Arzu Y, Can Cemal C, et al. The "physician on call patient engagement trial" (POPET): measuring the impact of a mobile patient engagement application on health outcomes and quality of life in allergic rhinitis and asthma patients. Int Forum Allergy Rhinol. 2015;5(6):487-497. doi:10.1002/alr.21468

\section{Publish your work in this journal}

Patient Preference and Adherence is an international, peer-reviewed, open access journal that focusing on the growing importance of patient preference and adherence throughout the therapeutic continuum. Patient satisfaction, acceptability, quality of life, compliance, persistence and their role in developing new therapeutic modalities and compounds to optimize clinical outcomes for existing disease states are major areas of interest for the journal. This journal has been accepted for indexing on PubMed Central. The manuscript management system is completely online and includes a very quick and fair peer-review system, which is all easy to use. Visit http:// www.dovepress.com/testimonials.php to read real quotes from published authors. 